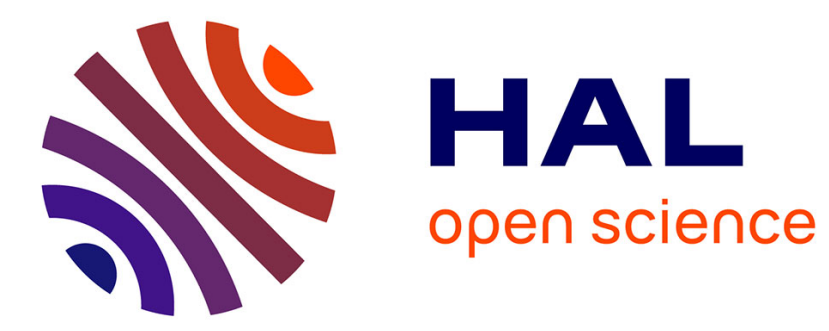

\title{
Linear and 2-frugal choosability of graphs of small maximum average degree
}

\author{
Nathann Cohen, Frédéric Havet
}

\section{To cite this version:}

Nathann Cohen, Frédéric Havet. Linear and 2-frugal choosability of graphs of small maximum average degree. [Research Report] RR-7213, INRIA. 2010. inria-00459692

\section{HAL Id: inria-00459692 \\ https://hal.inria.fr/inria-00459692}

Submitted on 24 Feb 2010

HAL is a multi-disciplinary open access archive for the deposit and dissemination of scientific research documents, whether they are published or not. The documents may come from teaching and research institutions in France or abroad, or from public or private research centers.
L'archive ouverte pluridisciplinaire HAL, est destinée au dépôt et à la diffusion de documents scientifiques de niveau recherche, publiés ou non, émanant des établissements d'enseignement et de recherche français ou étrangers, des laboratoires publics ou privés. 


\section{N R I A}

INSTITUT NATIONAL DE RECHERCHE EN INFORMATIQUE ET EN AUTOMATIQUE

Choisissabilité linéaire et 2-frugale des graphes de petit degré moyen maximum

N. Cohen - F. Havet

$\mathbf{N}^{\circ} \mathbf{7 2 1 3}$

24 février 2010

Thème COM

apport

de recherche 



\title{
Choisissabilité linéaire et 2-frugale des graphes de petit degré moyen maximum
}

\author{
N. Cohen , F. Havet \\ Thème COM — Systèmes communicants \\ Projets Mascotte \\ Rapport de recherche $\mathrm{n}^{\circ} 7213-24$ février $2010-21$ pages
}

\begin{abstract}
Résumé : Une coloration propre d'un graphe $G$ est 2-frugale (resp. linéaire) si le graphe induit par les sommets de toute paire de classes de couleurs est de degré maximum 2 (resp. est une forêt de chemins). Un graphe $G$ est 2-frugalement (linéairement) L-colorable si pour une assignation de listes $L: V(G) \mapsto 2^{\mathbb{N}}$ donnée, il existe une coloration 2-frugale (resp. linéaire) $c$ de $G$ telle que $c(v) \in L(v)$ pour tout sommet $v$ de $G$. Si $G$ est 2-frugalement (resp. linéairement) $L$-colorable pour toute assignation $L$ de listes telles que $|L(v)| \geq k$ pour tout sommet $v$ de $G$, ce graphe est alors dit 2-frugalement (resp. linéairement) choisissable. Dans cet article, nous améliorons des bornes connues sur la choisissabilité 2-frugale et la choisissabilité linéaire des graphes de petit degré moyen maximum.
\end{abstract}

Mots-clés : degré moyen maximum, coloration des sommets, coloration par liste, frugalité, coloration linéaire 


\title{
Linear and 2-frugal choosability of graphs of small maximum average degree
}

\begin{abstract}
A proper vertex colouring of a graph $G$ is 2-frugal (resp. linear) if the graph induced by the vertices of any two colour classes is of maximum degree 2 (resp. is a forest of paths). A graph $G$ is 2-frugally (resp. linearly) L-colourable if for a given list assignment $L: V(G) \mapsto 2^{\mathbb{N}}$, there exists a 2-frugal (resp. linear) colouring $c$ of $G$ such that $c(v) \in L(v)$ for all $v \in V(G)$. If $G$ is 2-frugally (resp. linearly) $L$-list colourable for any list assignment such that $|L(v)| \geq k$ for all $v \in V(G)$, then $G$ is 2-frugally (resp. linearly) $k$-choosable. In this paper, we improve some bounds on the 2-frugal choosability and linear choosability of graphs with small maximum average degree.
\end{abstract}

Key-words: maximum average degree, vertex-colouring, list colouring, frugality, linear coloring 


\title{
Linear and 2-frugal choosability of graphs of small maximum average degree
}

\author{
Nathann Cohen*, Frédéric Havet
}

A proper vertex colouring of a graph $G$ is 2-frugal (resp. linear) if the graph induced by the vertices of any two colour classes is of maximum degree 2 (resp. is a forest of paths). A graph $G$ is 2-frugally (resp. linearly) L-colourable if for a given list assignment $L: V(G) \mapsto 2^{\mathbb{N}}$, there exists a 2-frugal (resp. linear) colouring $c$ of $G$ such that $c(v) \in L(v)$ for all $v \in V(G)$. If $G$ is 2-frugally (resp. linearly) $L$-list colourable for any list assignment such that $|L(v)| \geq k$ for all $v \in V(G)$, then $G$ is 2-frugally (resp. linearly) $k$-choosable. In this paper, we improve some bounds on the 2-frugal choosability and linear choosability of graphs with small maximum average degree.

\section{Introduction}

The notion of acyclic colouring was introduced by Grünbaum [2] in 1973 : a vertex colouring is acyclic if it is proper (no two adjacent vertices have the same colour), and if there is no bicoloured cycle (the subgraph induced by the union of any two colours classes is a forest). A colouring such that for every vertex $v \in V(G)$, no colour appears more than $p$ times in the neighborhood of $v$, is said to be p-frugal, a notion introduced by Hind, Molloy and Reed in [3]. The p-frugal chromatic number of a graph $G$, denoted by $\Phi_{p}(G)$, is the minimum number of colours in a $p$ frugal colouring of $G$ and is clearly larger than $\left\lceil\frac{\Delta}{p}\right\rceil+1$. Hind, Molloy, and Reed [3] proved that $\Phi_{p}(G) \leq \max \left(p \Delta(G), \frac{e^{3}}{p} \Delta(G)^{1+1 / p}\right)$. In addition, they show that this upper bound is tight up to within a constant factor by showing graphs $G$ such that $\Phi_{p}(G) \geq \frac{1}{2 p} \Delta(G)^{1+1 / p}$.

Yuster [4] mixed the notions of 2-frugality and acyclicity, thus introducing the concept of linear colouring. A linear colouring of a graph is an acyclic and 2-frugal colouring. It can also be seen as a colouring such that the subgraph induced by the union of any two colour classes is a forest of paths (an acyclic graph with maximum degree at most two). The linear chromatic number of a graph $G$, denoted by $\Lambda[5(G)$, is the minimum number of colours in a linear colouring of $G$. As a linear colouring is 2-frugal, $\Lambda(G) \geq \Phi_{2}(G) \geq\left\lceil\frac{\Delta}{2}\right\rceil+1$. Yuster proved in [4] that $\Lambda(G)=O\left(\Delta(G)^{3 / 2}\right)$ in the general case, and he constructed graphs for which $\Lambda(G)=\Omega\left(\Delta(G)^{3 / 2}\right)$.

These concepts may be generalized to list colouring. Given a list assignment $L: V(G) \mapsto 2^{\mathbb{N}}$, an $L$-colouring of $G$ is a colouring $c$ such that $c(v) \in L(v)$ for each vertex $v$. A graph $G$ is $p$-frugally (resp. linearly) $L$-colourable if there is an $L$-colouring of $G$ which is $p$-frugal (resp. linear). If $G$ is 
$p$-frugally (resp. linearly) $L$-colourable for any assignment $L$ verifying $\forall v \in V(G),|L(v)| \geq k$, then $G$ is said to be $p$-frugally $k$-choosable (resp. linearly $k$-choosable). The smallest integer $k$ such that the graph $G$ is $p$-frugally $k$-choosable is the $p$-frugal choosability or $p$-frugal list chromatic number of $G$ and is denoted by $\Phi_{p}^{l}(G)$. The linear choosability or linear list chromatic number denoted $\Lambda^{l}(G)$ is defined analogously. The average degree of $G$ is $A d(G)=\frac{1}{|V(G)|} \sum_{v \in V(G)} d(v)=\frac{2|E(G)|}{|V(G)|}$. The maximum average degree of $G$ is $\operatorname{Mad}(G)=\max \{\operatorname{Ad}(H) \mid H$ is a subgraph of $G\}$.

In [1], Esperet et al. proved some upper bounds on the linear choosability of graphs with small maximum average degree.

Theorem 1 (Esperet et al. [1]). Let $G$ be a graph with maximum degree at most $\Delta$ :

1. If $\Delta \geq 3$ and $\operatorname{Mad}(G)<\frac{16}{7}$, then $\Lambda^{l}(G)=\left\lceil\frac{\Delta}{2}\right\rceil+1$.

2. If $\operatorname{Mad}(G)<\frac{5}{2}$, then $\Lambda^{l}(G) \leq\left\lceil\frac{\Delta}{2}\right\rceil+2$.

3. If $\operatorname{Mad}(G)<\frac{8}{3}$, then $\Lambda^{l}(G) \leq\left\lceil\frac{\Delta}{2}\right\rceil+3$.

In this article, we show in Theorem. 2 that the above upper bounds on the maximum average degree may be assumed arbitrarily close to 3 when $\Delta$ is large enough. When $\Delta$ is small, we also improve some of the existing bounds (see Theorem. 3). Since a linear colouring is 2 -frugal, the results are also valid for 2-frugal choosability. However, since being 2-frugal is less restrictive than being linear, we improve some of them in this case (see Theorem. 47. All these results, added to those proved by Esperet et al. [1] which have not been improved, are summarized in the following table :

\begin{tabular}{|c|c|c|c|}
\hline $\operatorname{Mad}(G)$ & $\Delta$ & $\Lambda^{l}(G)$ & \\
\hline$<\frac{16}{7} \approx 2.2857$ & $\geq 3$ & $\leq\left\lceil\frac{\Delta}{2}\right\rceil+1$ & Esperet et al. [1] \\
\hline$<\frac{39}{16}=2.4375$ & $\geq 5$ & $\leq\left\lceil\frac{\Delta}{2}\right\rceil+1$ & Theorem 31 \\
\hline$<\frac{48}{19} \approx 2.5263$ & $\geq 7$ & $\leq\left\lceil\frac{\Delta}{2}\right\rceil+1$ & Theorem 32 \\
\hline$<3-\frac{3}{\Delta+1}$ & $\geq 8$ & $\leq\left\lceil\frac{\Delta}{2}\right\rceil+1$ & Theorem 2 | \\
\hline & & $\leq\left\lceil\frac{\Delta}{2}\right\rceil+2$ & Esperet et al. [1] \\
\hline$<\frac{60}{23} \approx 2.6086$ & $\geq 5$ & $\leq\left\lceil\frac{\Delta}{2}\right\rceil+2$ & Theorem 33 \\
\hline$<3-\frac{9}{4 \Delta+3}$ & $\geq 7$ & $\leq\left\lceil\frac{\Delta}{2}\right\rceil+2$ & Theorem 2 2 \\
\hline$<\frac{14}{5}=2.8$ & & $\leq\left\lceil\frac{\Delta}{2}\right\rceil+3$ & Theorem 3 年 \\
\hline$<3$ & $\geq 12$ & $\leq\left\lceil\frac{\Delta}{2}\right\rceil+3$ & Theorem 23 \\
\hline$<3$ & & $\leq\left\lceil\frac{\Delta}{2}\right\rceil+4$ & Theorem 35 \\
\hline $\operatorname{Mad}(G)$ & $\Phi_{2}^{l}(G)$ & $\Delta$ & \\
\hline$<\frac{5}{2}$ & $\leq\left\lceil\frac{\Delta}{2}\right\rceil+1$ & $\geq 7$ & Theorem 41 \\
\hline$<3$ & $\leq\left\lceil\frac{\Delta}{2}\right\rceil+3$ & & Theorem 42 \\
\hline
\end{tabular}


The girth $g(G)$ of a graph $G$ is the length of its smallest cycle or $+\infty$ if $G$ has no cycle. Euler's formula implies that a planar graph $G$ has bounded maximum average degree in terms of its girth :

$$
\operatorname{Mad}(G)<2+\frac{4}{g(G)-2} .
$$

This immediately gives to any result on graphs with bounded maximum average degree have an equivalent formulation for planar graphs with large girth. These are summarized in the following table together with those coming from the papers of Esperet et al. [1] and Raspaud and Wang [5, 6] which are not improved here.

\begin{tabular}{|l|l|l|l|}
\hline girth & $\Lambda^{l}(G)$ & $\Delta$ & \\
\hline$\geq 16$ & $\leq\left\lceil\frac{\Delta}{2}\right\rceil+1$ & $\geq 3$ & Esperet et al. [1] \\
$\geq 7$ & $\leq\left\lceil\frac{\Delta}{2}\right\rceil+1$ & $\geq 13$ & Raspaud and Wang [5] \\
$\geq 8$ & $\leq\left\lceil\frac{\Delta}{2}\right\rceil+1$ & & \\
$\geq 10$ & $\leq\left\lceil\frac{\Delta}{2}\right\rceil+2$ & & Esperet et al \\
$\geq 9$ & $\leq\left\lceil\frac{\Delta}{2}\right\rceil+2$ & $\geq 5$ & \\
$\geq 7$ & $\leq\left\lceil\frac{\Delta}{2}\right\rceil+3$ & & \\
$\geq 6$ & $\leq\left\lceil\frac{\Delta}{2}\right\rceil+4$ & & \\
$\geq 5$ & $\leq\left\lceil\frac{\Delta}{2}\right\rceil+6$ & & Raspaud and Wang [6] \\
& $\leq\left\lceil\frac{9}{\Delta} 10\right\rceil+5$ & $\geq 85$ & Raspaud and Wang [6] \\
\hline \hline girth & $\Phi_{2}^{l}(G)$ & $\Delta$ & \\
\hline$\geq 6$ & $\leq\left\lceil\frac{\Delta}{2}\right\rceil+3$ & & \\
\hline
\end{tabular}

The proofs of our results are based on the same general idea. We study graphs which we call $k$-frugal-minimal (resp. $k$-linear-minimal) - i.e. graphs that are not $k$-frugally colourable (resp. $k$ linear-colourable), while any of their proper subgraphs is. We first show in Section 2 that some configurations (i.e. subgraphs) may not appear in such a graph. We then use in Section 3 the discharging method to show that a graph containing none of these forbidden configurations must have larger average degree than assumed, giving a contradiction .

\section{Forbidden configurations}

Before establishing some lemmas, let us give some definitions. Let $k$ a non-negative integer. A $k$-vertex (resp. $(\geq k)$-vertex, $(\leq k)$-vertex) is a vertex of degree exactly $k$ (resp. at least $k$, at most $k$ ). A $k$-neighbour of $v$ is a $k$-vertex adjacent to $v .(\geq k)$ and $(\leq k)$-neighbours are defined similarly. A $k$-thread in a graph $G$ is an induced path of $G$ with $k+1$ edges, and so $k$ internal vertices of degree 2 . 
Note that a $k$-frugal-minimal or $k$-linear-minimal graph is connected and in particular has no 0 -vertex. We will sometimes use this easy fact without referring explicitly to it.

\subsection{Linear colouring}

Lemma 1. Let $H$ be a $k$-linear-minimal or k-frugal-minimal graph.

1. If $k \geq\left\lceil\frac{\Delta(H)}{2}\right\rceil+1$, then $H$ has no 1-vertex.

2. For every 2-vertex $v$ with $N(v)=\{a, b\}$ and $\operatorname{deg}(a) \leq \operatorname{deg}(b)$, we have $\operatorname{deg}(b) \geq 2(k-\operatorname{deg}(a))+1$.

3. If $k \geq 3$, then $H$ contains no 3-thread.

4. If $k \geq 4$, then no 3-vertex is incident to a 2-thread.

5. Assume $k \geq 4$. If a 3-vertex has three 2-neighbours, then the second neighbour of each of those is a $(\geq 4)$-vertex.

6. If $k \geq 4$, then no 4-vertex is adjacent to four 2-threads.

7. If $k \geq 5$, then no 4-vertex is incident to a 2-thread.

8. If $k \geq 5$, then no 5-vertex is incident to five 2-threads.

9. If $k \geq 5$, then a 2-vertex has at most one 3-neighbour.

10. If $k \geq 5$, then a 4-vertex is not adjacent to two 2-vertices having each a 3-neighbour.

11. If $k \geq\left\lceil\frac{\Delta(H)}{2}\right\rceil+2$, then two 2-vertices are not adjacent.

12. If $k \geq\left\lceil\frac{\Delta(H)}{2}\right\rceil+3$, then every 3-vertex has no 2-neighbour.

13. If $k \geq\left\lceil\frac{\Delta(H)}{2}\right\rceil+3$, then every 4-vertex has at most three 2-neighbours.

14. If $k \geq\left\lceil\frac{\Delta(H)}{2}\right\rceil+4$, then every 4-vertex has at most four 2-neighbours.

15. If $k \geq \max \left(6,\left\lceil\frac{\Delta(H)}{2}\right\rceil+4\right)$, then every 5-vertex has at most two 2-neighbours.

Démonstration. In the following, we only prove the assertions for linear colouring : 2-frugal colouring being less restrictive, all the proofs translate naturally. Suppose that one of the assertions of Lemma 1 does not hold. Let $H$ be a $k$-linear-minimal graph for which it fails and $L$ a $k$-list assignment such that $H$ is not linearly $L$-colourable.

$11 H$ contains a 1 -vertex $u$. Let $v$ be the neighbour of $u$. Let $c$ be a linear $L$-colouring of $H-u$. We now extend $c$ to $u$. The colour $c(v)$ is forbidden. Moreover to preserve the 2 -frugality at $v$, the colours appearing twice in the neighbourhood of $v$ are also forbidden. There are at most $\left\lfloor\frac{\Delta(H)-1}{2}\right\rfloor$ such colours. Hence, at most $\left\lceil\frac{\Delta(H)}{2}\right\rceil$ colours in total are forbidden at $u$. Thus $u$ can be coloured with a non-forbidden colour in its list $L(u)$, and the colouring obtained is a linear $L$-colouring of $H$, which is a contradiction.

2 Let $v$ be a 2-vertex of $H$ with $N(v)=\{a, b\}$ and $\operatorname{deg}(a) \leq \operatorname{deg}(b)$, such that $\operatorname{deg}(b)<2(k-$ $\operatorname{deg}(a))+1$. Let $c$ be a linear $L$-colouring of $H-v$. 
- If $c(a)=c(b)$, let us assign to $v$ a colour $c(v) \in L(v)$ different from the ones of the other neighbours of $a$ (i.e. there are at most $\operatorname{deg}(a)-1$ of them), $c(a)$, and every colour which is repeated at least twice in the neighbourhood of $b$ (i.e. there at most $\left\lfloor\frac{\operatorname{deg}(b)-1}{2}\right\rfloor<k-\operatorname{deg}(a)$ of them). Doing so, we obtain a $k$-linear $L$-colouring of $H$, a contradiction.

- If $c(a) \neq c(b)$, let us assign to $v$ a colour $c(v) \in L(v)$ different from $c(a), c(b)$, and every colour which is repeated at least twice in the neighbourhood of $a$ or in the neighbourhood of $b$. The number of forbidden colours is at most $2+\left\lfloor\frac{\operatorname{deg}(a)-1}{2}\right\rfloor+\left\lfloor\frac{\operatorname{deg}(b)-1}{2}\right\rfloor<2+\operatorname{deg}(a)-2+k-$ $\operatorname{deg}(a)=k$ because $\operatorname{deg}(a) \geq 2$ and so $\operatorname{deg}(a) \geq 2+\left\lfloor\frac{\operatorname{deg}(a)-1}{2}\right\rfloor$. Hence such an assignment is possible, and yields a $k$-linear $L$-colouring of $H$, a contradiction.

3. It follows directly from 2 which implies that if a 2-vertex has a 2-neighbour then its other neighbour has degree at least 3 .

4 There is a configuration as depicted in Figure 1. with possibly $x=u_{1}$, or $x=u$ and $w=u_{1}$.

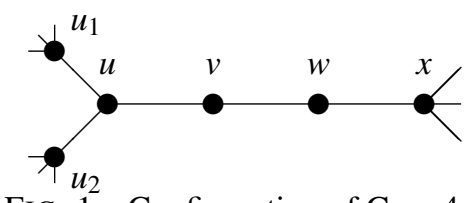

FIG. 1 - Configuration of Case 4

By the minimality of $H$, there exists a linear $L$-colouring $c$ of $H-v$. We now extend it to $v$ :

- If $c\left(u_{1}\right)=c\left(u_{2}\right)$, we colour $v$ with $c(v) \in L(v) \backslash\left\{c(u), c(w), c\left(u_{1}\right)\right\}$. There can be no bicoloured cycle, as $c(v)$ is different from both $c\left(u_{1}\right)$ and $c\left(u_{2}\right)$, and the 2-frugality at $u$ is preserved.

- If $c\left(u_{1}\right) \neq c\left(v_{2}\right)$, we colour $v$ with $c(v) \in L(v) \backslash\{c(u), c(w), c(x)\}$. There can be no bicoloured cycle, as $c(v)$ is different from $c(x)$, and $c$ is 2-frugal.

5 There is a configuration as depicted in Figure 2 Possibly $t_{i}=u_{i}$ and $w_{i}=u_{i}$ for $i=1$, 2, or some vertices in $\left\{w_{1}, w_{2}, t_{1}, t_{2}\right\}$ are identified.

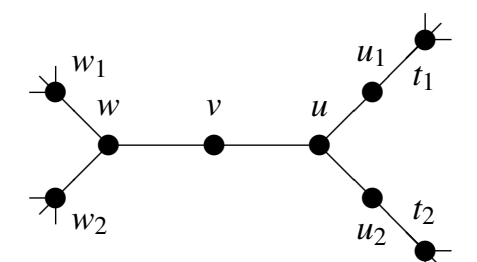

FIG. 2 - Configuration of Case 5

By the minimality of $H$ there exists a linear $L$-colouring $c$ of $H-\{u, v\}$. We now extend it to $u$ and $v$ :

- If $c\left(u_{1}\right)=c\left(u_{2}\right)$, we colour $v$ with $c(v) \in L(v) \backslash\left\{c(w), c\left(w_{1}\right), c\left(u_{1}\right)\right\}$ and $u$ with $c(u) \in L(v) \backslash\left\{c(v), c\left(u_{1}\right), c\left(t_{1}\right)\right\}$.

- If $c\left(u_{1}\right) \neq c\left(u_{2}\right)$, we colour $u$ with $c(u) \in L(u) \backslash\left\{c\left(u_{1}\right), c\left(u_{2}\right), c(w)\right\}$ and $v$ with $c(v) \in L(v) \backslash\left\{c(u), c(w), c\left(w_{1}\right)\right\}$.

6 There is a configuration as depicted in Figure 3 . Possibly $t_{3}=u=u_{3}$ and $u_{1}=t_{2}$ and $t_{1}=u_{2}$.

In this case, a linear colouring of $H-\left\{u_{1}, u_{2}\right\}$ can be extended into a linear colouring of $H$ by 
assigning to $u_{1}$ a colour distinct from those of $u$ and $w_{1}$ and to $u_{2}$ a colour distinct from those of $u, u_{1}$ and $v_{1}$. This is a contradiction.

So we may assume that it is not the case. Then possibly some vertices of $\left\{t_{3}, u_{3}, v_{3}, w_{3}\right\}$ may be identified.

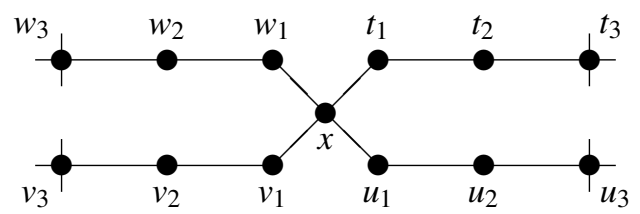

FIG. 3 - Configuration of Case 6

By the minimality of $H$ there exists a linear $L$-colouring $c$ of $H-\left\{t_{1}, u_{1}, v_{1}, w_{1}, x\right\}$. We now extend it to $t_{1}, u_{1}, v_{1}, w_{1}$ and $x$ :

- We colour $t_{1}$ with $c\left(t_{1}\right) \in L\left(t_{1}\right) \backslash\left\{c\left(t_{2}\right), c\left(t_{3}\right)\right\}$.

- We colour $u_{1}$ with $c\left(u_{1}\right) \in L\left(u_{1}\right) \backslash\left\{c\left(u_{2}\right), c\left(u_{3}\right), c\left(t_{1}\right)\right\}$.

- We colour $x$ with $c(x) \in L(x) \backslash\left\{c\left(t_{1}\right), c\left(u_{1}\right), c\left(w_{2}\right)\right\}$.

- We colour $w_{1}$ with $c\left(w_{1}\right) \in L\left(w_{1}\right) \backslash\left\{c\left(t_{2}\right), c(x)\right\}$.

- We colour $v_{1}$ with $c\left(v_{1}\right) \in L\left(v_{1}\right) \backslash\left\{c\left(v_{2}\right), c(x), c\left(w_{1}\right)\right\}$.

There can be no bicoloured cycle through $t_{1}$ (resp. $u_{1}$ ) as its colour is different from $c\left(t_{3}\right)$ (resp. $c\left(u_{3}\right)$ ), and none going through $w_{2}$ as its colour is different from $c(x)$.

7 There is a configuration as depicted in Figure 4. Possibly $w_{3}$ is one of $\{t, u, v\}$ or $w_{3}=x$ and $w_{2}=t$. In the later case, we get a contradiction as in Case 6, so we may assume that it does not occur.

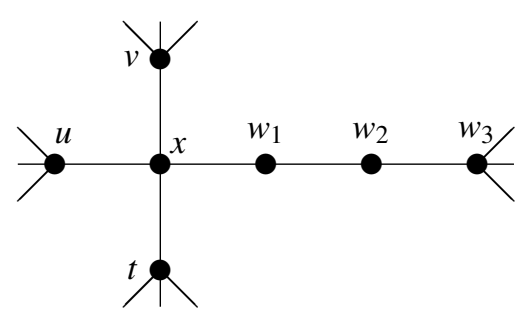

FIG. 4 - Configuration of Case 7

By the minimality of $H$ there exists a linear $L$-colouring $c$ of $H-w_{1}$. We extend it to $w_{1}$ :

- If $c\left(w_{2}\right)=c(x)$, then we colour $w_{1}$ with $c\left(w_{1}\right) \in L\left(w_{1}\right) \backslash\left\{c(x), c\left(w_{3}\right), c(v), c(t)\right\}$. There can be no bicoloured cycle as $c\left(w_{1}\right) \neq c\left(w_{3}\right)$, and the 2-frugality at $x$ is preserved.

- If $c\left(w_{2}\right) \neq c(x)$, then we colour $w_{1}$ with $c\left(w_{1}\right) \in L\left(w_{1}\right) \backslash\left\{c(x), c\left(w_{2}\right), c(v), c(t)\right\}$. There can be no bicoloured cycle as $c\left(w_{2}\right) \neq c(x)$, and the 2-frugality at $x$ is preserved.

8. There is a configuration as depicted in Figure 5 Possibly, some of the vertices in $\left\{s_{3}, t_{3}, u_{3}, v_{3}, w_{3}\right\}$ are the same or identified with $x$ (by pairs). In the later case, without loss of generality, $s_{3}=x=t_{3}$, 
$s_{1}=t_{2}$ and $t_{1}=s_{2}$. Then, by minimality of $H$, there is a linear colouring $c$ if $H-\left\{s_{1}, s_{2}\right\}$. It can be extended by colouring $s_{1}$ with a colour $c\left(s_{1}\right)$ distinct from $c(x)$ and the possible colour appearing twice on $\left\{u_{1}, v_{1}, w_{1}\right\}$, and colouring $s_{2}$ with a colour $c\left(s_{2}\right)$ distinct from $c(x), c\left(s_{1}\right)$ and the possible colour appearing twice on $\left\{u_{1}, v_{1}, w_{1}\right\}$. Hence we may assume that this case does not appear.

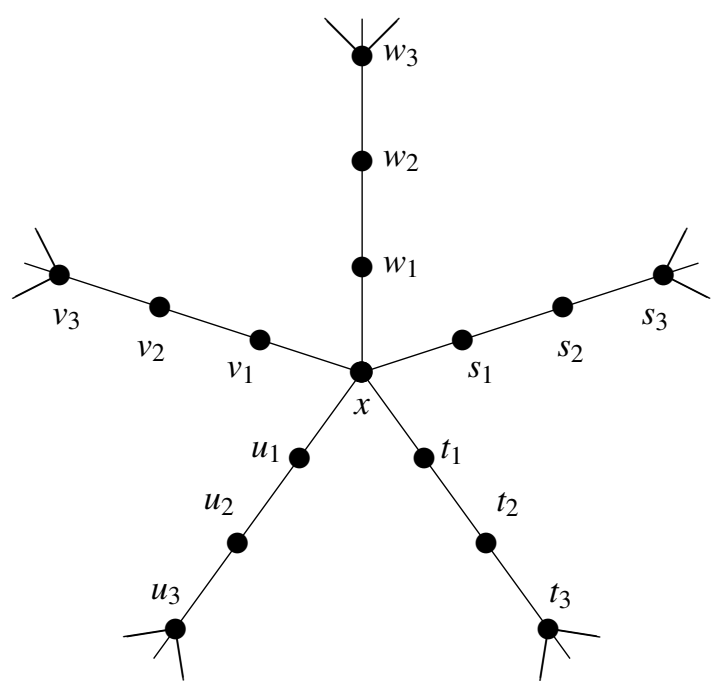

FIG. 5 - Configuration of Case 8

By the minimality of $H$ there exists a linear $L$-colouring $c$ of $H-\left\{x, s_{1}, t_{1}, u_{1}, v_{1}, w_{1}\right\}$. We extend it to $x, s_{1}, t_{1}, u_{1}, v_{1}$, and $w_{1}$ :

- We colour $s_{1}$ with $c\left(s_{1}\right) \in L\left(s_{1}\right) \backslash\left\{c\left(s_{2}\right), c\left(s_{3}\right)\right\}$.

- We colour $t_{1}$ with $c\left(t_{1}\right) \in L\left(t_{1}\right) \backslash\left\{c\left(t_{2}\right), c\left(t_{3}\right), c\left(s_{1}\right)\right\}$.

- We colour $u_{1}$ with $c\left(u_{1}\right) \in L\left(u_{1}\right) \backslash\left\{c\left(u_{2}\right), c\left(u_{3}\right), c\left(s_{1}\right), c\left(t_{1}\right)\right\}$.

- We colour $v_{1}$ with $c\left(v_{1}\right) \in L\left(v_{1}\right) \backslash\left\{c\left(v_{2}\right), c\left(u_{1}\right), c\left(t_{1}\right), c\left(s_{1}\right)\right\}$.

- We colour $x$ with $c(x) \in L(x) \backslash\left\{c\left(s_{1}\right), c\left(t_{1}\right), c\left(u_{1}\right), c\left(v_{1}\right)\right\}$.

- We colour $w_{1}$ with $c\left(w_{1}\right) \in L(w) \backslash\left\{c\left(w_{2}\right), c(x), c\left(v_{1}\right)\right\}$.

The 2-frugality at $x$ is preserved as 4 different colours are assigned to the vertices $s_{1}, t_{1}, u_{1}$ and $v_{1}$. Furthermore, there can be no bicoloured cycles going through $s_{1}$ and $s_{3}, t_{1}$ and $t_{3}, u_{1}$ and $u_{3}$ or $w_{1}$ and $v_{1}$. Thus the obtained $L$-colouring is linear, a contradiction.

9 There is a configuration as depicted in Figure 6 Possibly, some of the vertices of $\left\{v_{1}, v_{2}, w_{1}, w_{2}\right\}$ are identified or $v_{1}=v$ and $w_{1}=w$.

By the minimality of $H$, there exists a linear $L$-colouring $c$ of $H-u$. We extend it to $u$ :

- If $c(v)=c(w)$, we colour $u$ with $c(u) \in L(u) \backslash\left\{c(v), c\left(v_{1}\right), c\left(v_{2}\right), c\left(w_{1}\right)\right\}$ to prevent the formation of a bicoloured cycle and preserve the 2-frugality at $v$ and $w$.

- If $c(v) \neq c(w)$, we colour $u$ with $c(u) \in L(u) \backslash\left\{c(v), c\left(v_{1}\right), c\left(w_{1}\right), c(w)\right\}$ to preserve the 2frugality at $v$ and $w$. There can be no bicoloured cycles because $c(v) \neq c(w)$. 


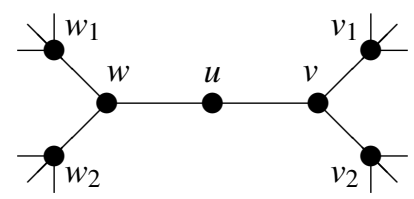

FIG. 6 - Configuration of Case 9

Hence $H$ is linearly $L$-colourable, a contradiction.

10. There is a configuration as depicted in Figure 7 Possibly some vertices of $\left\{v_{1}^{\prime \prime}, v_{1}^{\prime \prime \prime}, v_{2}^{\prime \prime}, v_{2}^{\prime \prime \prime}, v_{3}, v_{4}\right\}$ are the same, or, for $i \in\{1,2\}, v_{i}^{\prime \prime}=u$ and $v_{i}^{\prime}=v_{i+2}$.

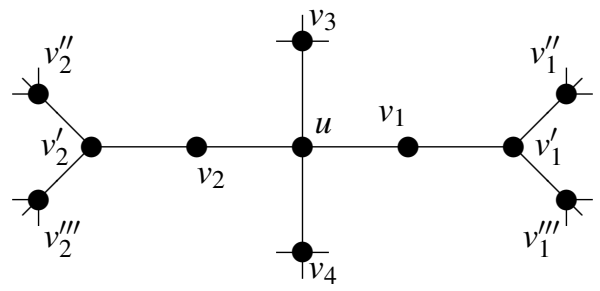

FIG. 7 - Configuration of Case 10

By the minimality of $H$ there exists a linear $L$-colouring $c$ of $H-\left\{v_{1}, v_{2}\right\}$. We extend it to $v_{1}$ and $v_{2}$ :

- If $c\left(v_{1}^{\prime}\right)=c(u)$, we colour $v_{1}$ with $c\left(v_{1}\right) \in L\left(v_{1}\right) \backslash\left\{c(u), c\left(v_{1}^{\prime \prime}\right), c\left(v_{1}^{\prime \prime \prime}\right), c\left(v_{4}\right)\right\}$. There can be no bicoloured cycle through $v_{1}$ as it is different from both $v_{1}^{\prime \prime}$ and $v_{1}^{\prime \prime \prime}$, and the 2-frugality at $v_{1}^{\prime}$ is preserved.

- If $c\left(v_{1}^{\prime}\right) \neq c(u)$, we colour $v_{1}$ with $c\left(v_{1}\right) \in L\left(v_{1}\right) \backslash\left\{c(u), c\left(v_{1}^{\prime}\right), c\left(v_{1}^{\prime \prime}\right), c\left(v_{4}\right)\right\}$. There can be no bicoloured cycle through $v_{1}$ as $c\left(v_{1}^{\prime}\right) \neq c(u)$, and the 2-frugality at $v_{1}^{\prime}$ is preserved.

We colour $v_{2}$ with symmetrical rules, replacing $v_{4}$ by $v_{3}$.

As $c\left(v_{4}\right) \neq c\left(v_{1}\right)$ and $c\left(v_{3}\right) \neq c\left(v_{2}\right)$, the 2-frugality of $c$ is preserved.

11. It follows from 2 Indeed if a 2-vertex would have a 2-neighbour then its second neighbour has degree at least $\Delta(H)+1$, a contradiction.

12. There is a configuration as depicted in Figure 8 with possibly $v_{1}^{\prime}=v_{2}$.

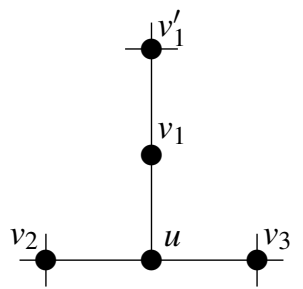

FIG. 8 - Configuration of Case 12

By the minimality of $H$, there exists a linear $L$-colouring $c$ of $H-v_{1}$. 
- If $c(u)=c\left(v_{1}^{\prime}\right)$, we colour $v_{1}$ with $c\left(v_{1}\right) \in L\left(v_{1}\right)$ that is different from $c(u)$, the colours appearing twice in the neighbourhood of $v_{1}^{\prime}$ (in order to preserve the 2-frugality at this vertex) and different from $c\left(v_{2}\right)$ and $c\left(v_{3}\right)$ to prevent the apparition of bicoloured cycles.

- If $c(u) \neq c\left(v_{1}^{\prime}\right)$, we colour $v_{1}$ with $c\left(v_{1}\right) \in L\left(v_{1}\right)$ that is different from $c(u)$ and $c\left(v_{1}^{\prime}\right)$, the colours appearing twice in the neighbourhood of $v_{1}^{\prime}$ (in order to preserve 2-frugality at this vertex) and different from $c\left(v_{2}\right)$ to preserve the 2-frugality at $u$. There can be no bicoloured cycles as $c(u) \neq c\left(v_{1}^{\prime}\right)$.

In both cases, $H$ is linearly $L$-colourable, a contradiction.

13. There is a configuration as depicted in Figure 9 with possibly some of the vertices in $\left\{v_{1}^{\prime}, v_{2}^{\prime}, v_{3}^{\prime}, v_{4}^{\prime}\right\}$ being identified.

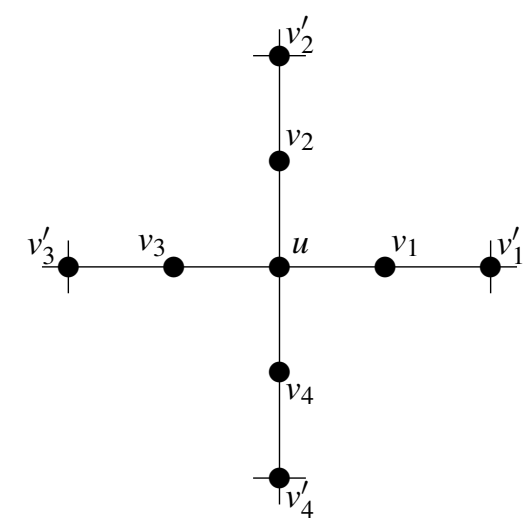

FIG. 9 - Configuration of Case 13

By the minimality of $H$ there exists a linear $L$-colouring $c$ of $H-\left\{u, v_{1}, v_{2}, v_{3}, v_{4}\right\}$. We extend it to $u, v_{1}, v_{2}, v_{3}$ and $v_{4}$ :

- We colour $u$ with $c(u) \in L(u) \backslash\left\{c\left(v_{1}^{\prime}\right), c\left(v_{2}^{\prime}\right), c\left(v_{3}^{\prime}\right), c\left(v_{4}^{\prime}\right)\right\}$ to prevent the apparition of bicoloured cycles.

- We colour $v_{1}$ with $c\left(v_{1}\right) \in L\left(v_{1}\right)$ different from $c(u), c\left(v_{1}^{\prime}\right)$ and the colours appearing twice in the neighbourhood of $v_{1}^{\prime}$.

- We colour $v_{2}$ with $c\left(v_{2}\right) \in L\left(v_{2}\right)$ different from $c(u), c\left(v_{2}^{\prime}\right)$ and the colours appearing twice in the neighbourhood of $v_{2}^{\prime}$.

- We colour $v_{3}$ with $c\left(v_{3}\right) \in L\left(v_{3}\right)$ different from $c(u), c\left(v_{3}^{\prime}\right), c\left(v_{2}\right)$ and the colours appearing twice in the neighbourhood of $v_{3}^{\prime}$.

- We colour $v_{4}$ with $c\left(v_{4}\right) \in L\left(v_{4}\right)$ different from $c(u), c\left(v_{4}^{\prime}\right), c\left(v_{1}\right)$ and the colours appearing twice in the neighbourhood of $v_{4}^{\prime}$.

There can be no bicoloured cycle with this colouring of $H$ and $c$ is 2-frugal because 3 vertices among $\left\{v_{1}, v_{2}, v_{3}, v_{4}\right\}$ cannot share the same colour. Then $H$ is linearly $L$-colourable, a contradiction.

14. There is a configuration as depicted in Figure 10 with possibly some of the vertices in $\left\{v_{1}^{\prime}, v_{2}^{\prime}, v_{3}^{\prime}, v_{4}\right\}$ being identified. 


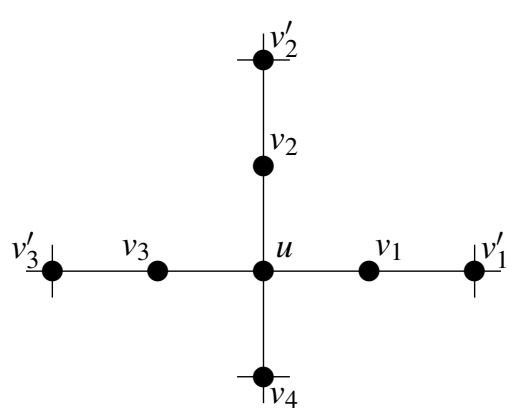

FIG. 10 - Configuration of Case 14

By the minimality of $H$ there exists a linear $L$-colouring $c$ of $H-\left\{u, v_{1}, v_{2}, v_{3}\right\}$. We extend it to $u$, $v_{1}, v_{2}$ and $v_{3}$ :

- We colour $u$ with $c(u) \in L(u)$ such that $c(u)$ is different $c\left(v_{4}\right), c\left(v_{1}^{\prime}\right), c\left(v_{2}^{\prime}\right), c\left(v_{3}^{\prime}\right)$ (to avoid any bicoloured cycle) and the colours appearing twice in the neighbourhood of $v_{4}$.

- We colour $v_{1}$ with $c\left(v_{1}\right) \in L\left(v_{1}\right)$ different from $c(u), c\left(v_{1}^{\prime}\right), c\left(v_{4}\right)$, and the colours appearing twice in the neighbourhood of $v_{1}^{\prime}$.

- We colour $v_{3}$ with $c\left(v_{3}\right) \in L\left(v_{3}\right)$ different from $c(u), c\left(v_{3}^{\prime}\right), c\left(v_{4}\right)$, and the colours appearing twice in the neighbourhood of $v_{3}^{\prime}$.

- We colour $v_{2}$ with $c\left(v_{2}\right) \in L\left(v_{2}\right)$ different from $c(u), c\left(v_{2}^{\prime}\right), c\left(v_{3}\right), c\left(v_{1}\right)$, and the colours appearing twice in the neighbourhood of $v_{2}^{\prime}$.

There is no bicoloured cycle containing $u$ because $c(u)$ is different from $c\left(v_{1}^{\prime}\right), c\left(v_{2}^{\prime}\right)$ and $c\left(v_{3}^{\prime}\right)$. Moreover, the 2-frugality at $U$ is assured, as 3 vertices among $\left\{v_{1}, v_{2}, v_{3}, v_{4}\right\}$ cannot share the same colour. Hence $H$ is linearly $L$-colourable, a contradiction.

15. There is a configuration as depicted in Figure 11 with possibly some of the vertices in $\left\{v_{1}^{\prime}, v_{2}^{\prime}, v_{3}^{\prime}, v_{4}^{\prime}, v_{5}^{\prime}\right\}$ being identified.

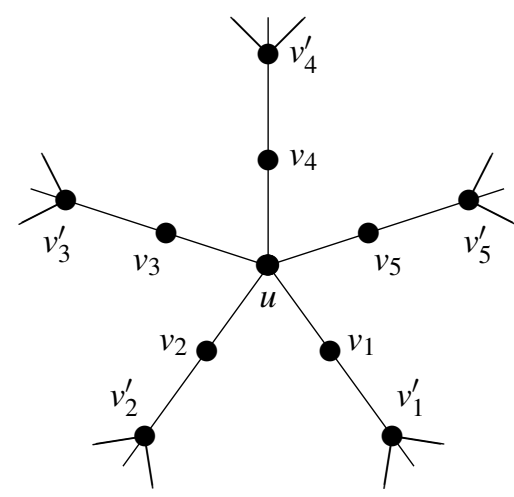

FIG. 11 - Configuration of Case 15

By the minimality of $H$ there exists a linear $L$-colouring $c$ of $H-\left\{u, v_{1}, v_{2}, v_{3}, v_{4}, v_{5}\right\}$. We extend it to $u, v_{1}, v_{2}, v_{3}, v_{4}$ and $v_{5}$ : 
- We colour $v_{1}$ with $c\left(v_{1}\right) \in L\left(v_{1}\right)$ different from $c\left(v_{1}^{\prime}\right)$, and the colours appearing twice in the neighbourhood of $v_{1}^{\prime}$.

- We colour $v_{2}$ with $c\left(v_{2}\right) \in L\left(v_{2}\right)$ different from $c\left(v_{2}^{\prime}\right), c\left(v_{1}\right)$, and the colours appearing twice in the neighbourhood of $v_{2}^{\prime}$.

- We colour $v_{3}$ with $c\left(v_{3}\right) \in L\left(v_{3}\right)$ different from $c\left(v_{3}^{\prime}\right), c\left(v_{1}\right), c\left(v_{2}\right)$, and the colours appearing twice in the neighbourhood of $v_{3}^{\prime}$.

- We colour $v_{5}$ with $c\left(v_{5}\right) \in L\left(v_{5}\right)$ different from $c\left(v_{5}^{\prime}\right), c\left(v_{1}\right), c\left(v_{2}\right), c\left(v_{3}\right)$ and the colours appearing twice in the neighbourhood of $v_{5}^{\prime}$.

- We colour $u$ with $c(u) \in L(u)$ different from $c\left(v_{1}\right), c\left(v_{2}\right), c\left(v_{3}\right), c\left(v_{5}\right)$, and $c\left(v_{4}^{\prime}\right)$.

- We colour $v_{4}$ with $c\left(v_{4}\right) \in L\left(v_{4}\right)$ different from $c\left(v_{4}^{\prime}\right), c(u)$, and the colours appearing twice in the neighbourhood of $v_{4}^{\prime}$.

There is no bicoloured cycle using $v_{4}^{\prime}$ because $c\left(v_{4}^{\prime}\right) \neq c(u)$. Moreover there is no bicoloured cycle using both $v_{i}$ and $v_{j}$, for $i<j$ and $i, j \in\{1,2,3,5\}$ as $c\left(v_{1}\right), c\left(v_{2}\right), c\left(v_{3}\right)$ and $c\left(v_{5}\right)$ are all distionct. For the same reason, the 2-frugality at $u$ is assured. Thus $H$ is linearly $L$-colourable, a contradiction.

\subsection{2-frugal colouring}

Lemma 2. Let $H$ be a $k$-frugal-minimal graph.

(i) If $k \geq 4$, then no 4-vertex is incident to two 2-threads.

(ii) If $k \geq\left\lceil\frac{\Delta}{2}\right\rceil+3$, then a 4-vertex has at most one 2-neighbour.

(iii) If $k \geq \max \left(6,\left\lceil\frac{\Delta}{2}\right\rceil+3\right)$, then a 5-vertex has at most four 2-neighbour.

Démonstration. Suppose that one of the assertions of Lemma2 does not hold. Let $H$ be a $k$-frugalminimal graph for which it fails and $L$ a $k$-list assignment such that $H$ is not 2-frugally $L$-colourable.

(i) There is a configuration as depicted in Figure 12

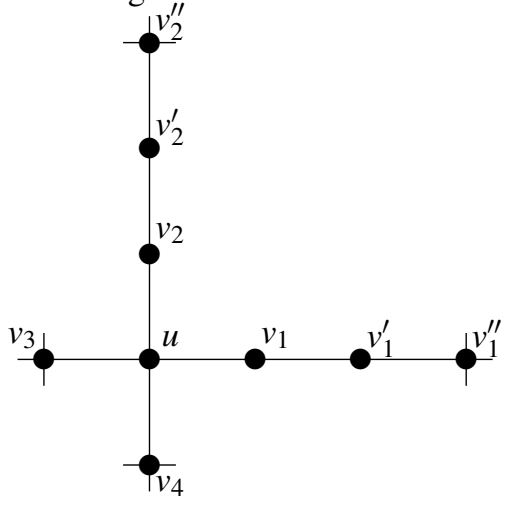

FIG. 12 - Configuration of Case (ii)

By the minimality of $H$ there exists a 2-frugal $L$-colouring $c$ of $H-\left\{v_{1}, v_{2}\right\}$. We extend it to $v_{1}$ and $v_{2}$ : 
- We colour $v_{1}$ with $c\left(v_{1}\right) \in L\left(v_{1}\right)$ different from $c(u), c\left(v_{4}\right), c\left(v_{1}^{\prime}\right)$.

- We colour $v_{2}$ with $c\left(v_{2}\right) \in L\left(v_{2}\right)$ different from $c(u), c\left(v_{3}\right), c\left(v_{2}^{\prime}\right)$.

$c$ is 2-frugal, given that $c\left(v_{1}\right) \neq c\left(v_{4}\right)$ and $c\left(v_{2}\right) \neq c\left(v_{3}\right)$. Hence, $H$ is 2-frugally $L$-colourable, a contradiction.

(ii) There is a configuration as depicted in Figure 13 with possibly some of the vertices in $\left\{v_{1}^{\prime}, v_{2}^{\prime}, v_{3}, v_{4}\right\}$ being identified.

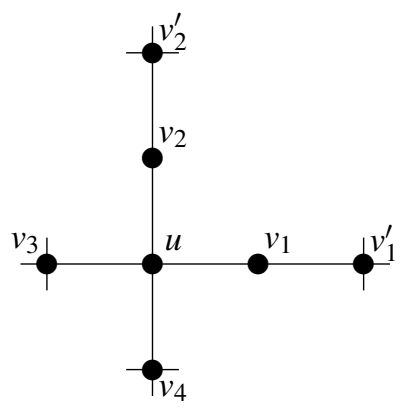

FIG. 13 - Configuration of Case (iii)

By the minimality of $H$ there exists a 2-frugal $L$-colouring $c$ of $H-\left\{v_{1}, v_{2}\right\}$. We extend it to $v_{1}$ and $v_{2}$ :

- We colour $v_{1}$ with $c\left(v_{1}\right) \in L\left(v_{1}\right)$ different from $c(u), c\left(v_{4}\right), c\left(v_{1}^{\prime}\right)$ and the colours appearing twice in the neighbourhood of $v_{1}^{\prime}$.

- We colour $v_{2}$ with $c\left(v_{2}\right) \in L\left(v_{2}\right)$ different from $c(u), c\left(v_{3}\right), c\left(v_{2}^{\prime}\right)$ and the colours appearing twice in the neighbourhood of $v_{2}^{\prime}$.

$c$ is 2-frugal, given that $c\left(v_{1}\right) \neq c\left(v_{4}\right)$ and $c\left(v_{2}\right) \neq c\left(v_{3}\right)$. Hence, $H$ is 2-frugally $L$-colourable, a contradiction.

(iii) There is a configuration as depicted in Figure 14 with possibly some vertices in $\left\{v_{1}^{\prime}, v_{2}^{\prime}, v_{3}^{\prime}, v_{4}^{\prime}, v_{5}^{\prime}\right\}$ being identified.
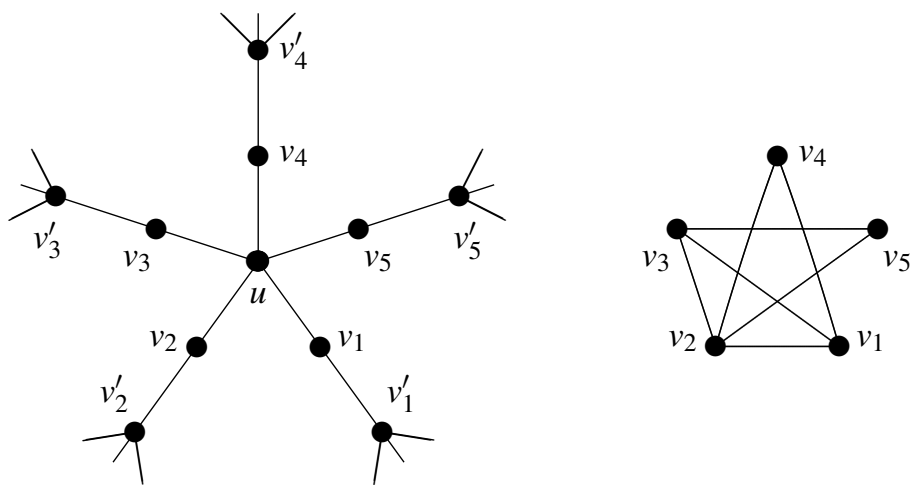

FIG. 14 - Configuration of Case (iv) and its auxiliary graph 
By the minimality of $H$ there exists a 2 -frugal $L$-colouring $c$ of $H-\left\{u, v_{1}, v_{2}, v_{3}, v_{4}, v_{5}\right\}$. We extend it to $u, v_{1}, v_{2}, v_{3}, v_{4}$ and $v_{5}$ :

- We colour $v_{1}$ with $c\left(v_{1}\right) \in L\left(v_{1}\right)$ different from $c\left(v_{1}^{\prime}\right)$, and the colours appearing twice in the neighbourhood of $v_{1}^{\prime}$.

- We colour $v_{2}$ with $c\left(v_{2}\right) \in L\left(v_{2}\right)$ different from $c\left(v_{2}^{\prime}\right), c\left(v_{1}\right)$ and the colours appearing twice in the neighbourhood of $v_{2}^{\prime}$.

- We colour $v_{3}$ with $c\left(v_{3}\right) \in L\left(v_{3}\right)$ different from $c\left(v_{3}^{\prime}\right), c\left(v_{1}\right), c\left(v_{2}\right)$ and the colours appearing twice in the neighbourhood of $v_{3}^{\prime}$.

- We colour $v_{4}$ with $c\left(v_{4}\right) \in L\left(v_{4}\right)$ different from $c\left(v_{4}^{\prime}\right), c\left(v_{1}\right), c\left(v_{2}\right)$ and the colours appearing twice in the neighbourhood of $v_{4}^{\prime}$.

- We colour $v_{5}$ with $c\left(v_{5}\right) \in L\left(v_{5}\right)$ different from $c\left(v_{5}^{\prime}\right), c\left(v_{2}\right), c\left(v_{3}\right)$ and the colours appearing twice in the neighbourhood of $v_{5}^{\prime}$.

- We colour $u$ with $c(u) \in L(u)$ different from $c\left(v_{1}\right), c\left(v_{2}\right), c\left(v_{3}\right), c\left(v_{4}\right)$ and $c\left(v_{5}\right)$.

The colouring is 2 -frugal at $u$ because no three vertices of $\left\{v_{1}, v_{2}, v_{3}, v_{4}, v_{5}\right\}$ can share the same colour as there is no stable set of size 3 in the auxiliary graph depicted on the right of Figure 14 Thus $H$ is 2-frugally $L$-colourable, a contradiction.

\section{Main results}

\subsection{Linear colouring - asymptotic result}

In this subsection, we prove the following theorem :

Theorem 2. Let $G$ be a graph of maximum degree at most $\Delta$.

1. If $\operatorname{Mad}(G)<3-\frac{3}{\Delta+1}$ and $\Delta \geq 8$, then $\Lambda^{l}(G) \leq\left\lceil\frac{\Delta}{2}\right\rceil+1$.

2. If $\operatorname{Mad}(G)<3-\frac{9}{4 \Delta+3}$ and $\Delta \geq 7$, then $\Lambda^{l}(G) \leq\left\lceil\frac{\Delta}{2}\right\rceil+2$.

3. If $\operatorname{Mad}(G)<3$ and $\Delta \geq 12$ then $\Lambda^{l}(G) \leq\left\lceil\frac{\Delta}{2}\right\rceil+3$.

Démonstration. The proof of the three statements are similar.

We assume the existence of a counter-example $G$ with maximum degree at most $\Delta$ such that $\operatorname{Mad}(G)<3-\varepsilon$ (we first consider $\varepsilon$ as a variable). $G$ then contains a subgraph $H$ which is $\left(\left\lceil\frac{\Delta}{2}\right\rceil+q\right)$ linear-minimal with $q=1,2$ and 3 depending one the statement. We give to each vertex $v$ of $H$ an initial charge $w(v)$ equal to its degree $\operatorname{deg}_{H}(v)$ in $H$. The average charge is then equal to the average degree of $H$ which is at most $\operatorname{Mad}(G)$.

We then use the following discharging rule :

- Every $d$-vertex, $d \geq 3$, gives $\alpha_{d}=\frac{d-(3-\varepsilon)}{d}$ to its 2-neighbours.

We shall prove that after the discharging phase every vertex $v$ has final charge $w^{*}(v)$ at least $3-\varepsilon$ for some $\varepsilon \geq 0$ to be determined. This implies that

$$
A d(H)=\frac{\sum_{v \in V(H)} w(v)}{|V(H)|}=\frac{\sum_{v \in V(H)} w^{*}(v)}{|V(H)|} \geq 3-\varepsilon
$$


which contradicts $\operatorname{Mad}(G)<3-\varepsilon$.

By Lemma 1 1, there is no $(\leq 1)$-vertex. For any $d \geq 3$, every $d$-vertex send at least $d$ times $\alpha_{d}$, so its final charge is at least $d-\bar{d} \cdot \alpha_{d}=3-\varepsilon$.

Let us now examine the final charge of 2-vertices. We set $d_{q}^{\prime}=2\left(\left\lceil\frac{\Delta}{2}\right\rceil+q-d\right)+1$. By Lemma 1 2. every 2-vertex having a $d$-neighbour has also a $\left(\geq d_{q}^{\prime}\right)$-neighbour. Observe that $d_{q}^{\prime}>\Delta$ if $d \leq q$, thus no 2 -vertex has a $(\leq q)$-neighbour.

If $q \geq 2$, then a 2-vertex $v$ has no 2-neighbour. Hence by Lemma 1, 2 , it has a $d_{1}$-neighbour and a $d_{2}$-neighbour, with $2<d=d_{1} \leq d_{q}^{\prime} \leq d_{2}$. Since $\alpha_{3} \leq \alpha_{4} \leq \cdots \leq \alpha_{\Delta}$, the final charge of $v$ is at least $w^{*}(v)=2+\alpha_{d_{1}}+\alpha_{d_{2}} \geq 2+\alpha_{d}+\alpha_{d_{q}^{\prime}}$.

If $q=1$ a 2 -vertex has either no 2 -neighbour and as above its final charge is at least $2+\alpha_{d}+\alpha_{d_{q}^{\prime}}$ or it has a 2-neighbour and, by Lemma 1 2, its other neighbour is a $\Delta$-vertex, so it final charge is at least $2+\alpha_{\Delta}$.

Hence, to prove Theorem 2 it is sufficient to show that $2+\alpha_{d}+\alpha_{d_{q}^{\prime}} \geq 3-\varepsilon$, for all $d \leq d_{q}^{\prime}$ and also $2+\alpha_{\Delta} \geq 3-\varepsilon$ when $q=1$.

$2+\alpha_{d}+\alpha_{d_{q}^{\prime}} \geq 3-\varepsilon$ is equivalent to $P(d) \geq 0$ with $P(d)=(1+\varepsilon) d d_{q}^{\prime}+(\varepsilon-3)\left(d+d_{q}^{\prime}\right)$. Since $d_{q}^{\prime}=2\left(\left\lceil\frac{\Delta}{2}\right\rceil+q-d\right)+1, P(d)$ is a polynomial of degree 2 in $d$ of the form $-2(1-\varepsilon) d^{2}+A \cdot d+B$ for some constant $A$ and $B$ (note that the coefficient of $d^{2}$ is negative). Hence to verify that $P(d) \geq 0$ for all possible values of $d$ it suffices to prove it for the smallest and largest $d$ such that $d \leq d_{q}^{\prime}$, namely $\max (3, q+1)$ and $\frac{\Delta+2 q+2}{3}$ (it actually is $\frac{\Delta+2 q+2}{3}$ if $\Delta$ is odd and $\frac{\Delta+2 q+1}{3}$ if $\Delta$ is even).

If $q \geq 2$, we obtain the following two conditions :

$$
\begin{aligned}
\varepsilon & \geq \frac{3(q+1)-(q-2) \Delta}{q+1+(q+2) \Delta} \\
\varepsilon & \geq \frac{16-2 q-\Delta}{8+2 q+\Delta}
\end{aligned}
$$

For $q=3$ and $\Delta \geq 12$ the right hand sides of these two inequalities are negative. So they are satisfied for $\varepsilon=0$, which proves Theorem 2 3 . For $q=2$ and $\Delta \geq 7$ then $\frac{9}{3+4 \Delta} \geq \frac{12-\Delta}{12+\Delta}$ and so the above inequalities are satisfied for $\varepsilon=\frac{9}{3+4 \Delta}$. This proves Theorem 2

If $q=1$, we have the three following conditions, the first two given by $P(d)$ and the third one by $2+\alpha_{\Delta} \geq 3-\varepsilon$.

$$
\begin{aligned}
& \varepsilon \geq \frac{9}{4 \Delta-5} \\
& \varepsilon \geq \frac{14-\Delta}{10+\Delta} \\
& \varepsilon \geq \frac{3}{\Delta+1}
\end{aligned}
$$

But $\frac{3}{\Delta+1} \geq \frac{9}{4 \Delta-5}$ and $\frac{3}{\Delta+1} \geq \frac{14-\Delta}{10+\Delta}$ when $\Delta \geq 8$. So the above inequalities are satisfied for $\varepsilon=\frac{3}{\Delta+1}$. This proves Theorem 2 , 1 . 


\subsection{Graphs of small maximum degree}

In this subsection we prove the following theorem.

Theorem 3. Let $G$ be a graph with maximum degree at most $\Delta$ :

1. If $\Delta \geq 5$ and $\operatorname{Mad}(G)<\frac{39}{16}$, then $\Lambda^{l}(G) \leq\left\lceil\frac{\Delta}{2}\right\rceil+1$.

2. If $\Delta \geq 7$ and $\operatorname{Mad}(G)<\frac{48}{19}$, then $\Lambda^{l}(G) \leq\left\lceil\frac{\Delta}{2}\right\rceil+1$.

3. If $\Delta \geq 5$ and $\operatorname{Mad}(G)<\frac{60}{23}$, then $\Lambda^{l}(G) \leq\left\lceil\frac{\Delta}{2}\right\rceil+2$.

4. If $\operatorname{Mad}(G)<\frac{14}{5}$, then $\Lambda^{l}(G) \leq\left\lceil\frac{\Delta}{2}\right\rceil+3$.

5. If $\operatorname{Mad}(G)<3$. Then $\Lambda^{l}(G) \leq\left\lceil\frac{\Delta}{2}\right\rceil+4$.

Démonstration. The proofs of all statements are similar : we assume the existence of a counterexample $G$ such that $\operatorname{Mad}(G)<M$ (we first consider $M$ as a variable), from which we deduce the existence of a subgraph $H$ which is $k$-linear-minimal. We then use the discharging method to reach a contradiction.

We give to each vertex $v$ of $H$ an initial charge $w(v)$ equal to its degree $\operatorname{deg}_{H}(v)$ in $H$. The average charge is then equal to the average degree of $H$ which is at most $\operatorname{Mad}(G)$. Then, we define discharging rules by which vertices will exchange some of their charge, keeping the average constant. We then want to prove with the help of the lemmas of the previous section that each vertex $v$ has a final charge $w^{*}(v)$ at least $M$ and so strictly greater than the average charge, which is a contradiction.

Of course, we want to find rules such that $M$ is as large as possible in each case : for this reason, the following proofs actually define the constraints of a Linear Programme in which $M$ is the objective value, and whose variables are the charges exchanged by the vertices during the discharging phase. At the end of each proof, we give an optimal solution of the given Linear Programme which proves the results.

1 Let $G$ be a graph with maximum degree $\Delta \geq 5$ such that $\operatorname{Mad}(G)<M$. Set $k_{1}=\left\lceil\frac{\Delta}{2}\right\rceil+1$. Suppose by way of contradiction that $\Lambda^{l}(G)>k_{1}$. Then $G$ has a subgraph $H$ which is $k_{1}$-linearminimal.

Let us assign to every vertex of $H$ an initial charge $w(v)=\operatorname{deg}_{H}(v)$. Then $\sum_{v \in V(H)} w(v)=$ $\sum_{v \in V(H)} d(v)=A d(H) \cdot|V(H)|$. We now apply the following discharging rules.

Rule 1. A 2-vertex having two 3-neighbours receives $\alpha_{3}$ from each of them.

Rule 2. A 2-vertex having only one 3-neighbour receives $\alpha_{3}^{\prime}$ from it.

Rule 3. A 2-vertex having a 2-neighbour and a ( $\geq 4)$-neighbour receives $\alpha_{4}$ from it.

Rule 4. A 2 -vertex having a $(\geq 3)$-neighbour and a $(\geq 4)$-neighbour receives $\alpha_{4}^{\prime}$ from its $(\geq 4)$-neighbour.

At the end we want that the final charge of every vertex is at least $M$. This implies

$$
A d(H)=\frac{\sum_{v \in V(H)} w(v)}{|V(H)|}=\frac{\sum_{v \in V(H)} w^{*}(v)}{|V(H)|} \geq M
$$

which contradicts $\operatorname{Mad}(G)<M$. 
We now define constraints on $M$ and the $\alpha_{i}$ and $\alpha_{i}^{\prime}$ guaranteeing the the final charge $w^{*}(v)$ of every vertex $v$ of $H$ is at least $M$.

As there are no $(\leq 1)$-vertices by Lemma|1 1 , let us examine the final charge $w^{*}(v)$ of a $(\geq 2)$ vertex $v$.

- If $v$ is a 2-vertex then by Lemma 1-3 and 14 it has either two 3-neighbours, or one 3-neighbour and one ( $\geq 4)$-neighbour, or one 2-neighbour and one ( $\geq 4)$-neighbour. In the first case $w^{*}(v)=$ $2+2 \alpha_{3}$, on the second $w^{*}(v) \leq 2+\alpha_{3}^{\prime}+\alpha_{4}^{\prime}$ and in the last one $w^{*}(v)=2+\alpha_{4}$. So the required constraints are

$$
M \leq 2+2 \alpha_{3} \text { and } M \leq 2+\alpha_{3}^{\prime}+\alpha_{4}^{\prime} \text { and } M \leq 2+\alpha_{4} .
$$

- If $v$ is a 3-vertex adjacent to three 2-neighbours, none of them can be adjacent to another 3vertex by Lemma 1,5 , and so will give at most $3 \alpha_{3}^{\prime}$. If it is adjacent to two 2-neighbours then it gives at most $\max \left\{2 \alpha_{3}, 2 \alpha_{3}^{\prime}\right\}$. Hence, we obtain the constraints

$$
M \leq 3-3 \alpha_{3}^{\prime} \text { and } M \leq 3-2 \alpha_{3} .
$$

- If $v$ is a 4-vertex, by Lemma 110 it can not be adjacent to four 2-threads. Then it gives at most $3 \alpha_{4}+\alpha_{4}^{\prime}$. (Here we assume implicitly that $\alpha_{4}^{\prime} \leq \alpha_{4}$ which is intuitively true but a priori not proved. However this inequality is satisfied by the solution giving the optimal value of $M$ and so our assumption is a posteriori correct). Thus we obtain the constraint $M \leq 4-3 \alpha_{4}-\alpha_{4}^{\prime}$.

- If $v$ is a $k$-vertex with $k \geq 5$, it will give at most give $k \alpha_{4}$ yielding the constraints $M \leq k-k \alpha_{4}$. The optimal value $M=\frac{39}{16}$ is obtained for $\alpha_{3}=\frac{7}{32}, \alpha_{3}^{\prime}=\frac{3}{16}, \alpha_{4}=\frac{7}{16}$ and $\alpha_{4}^{\prime}=\frac{1}{4}$.

2 Let $G$ be a graph with maximum degree $\Delta \geq 7$ such that $\operatorname{Mad}(G)<M$. Set $k_{1}=\left\lceil\frac{\Delta}{2}\right\rceil+1$. Suppose by way of contradiction that $\Lambda^{l}(G)>k_{1}$. Then $G$ has a subgraph $H$ which is $k_{1}$-linearminimal.

Let us assign to every vertex of $H$ an initial charge $w(v)=\operatorname{deg}_{H}(v)$ and apply the following discharging rules.

Rule 1. A 3-vertex sends $\alpha_{3}$ to each of its 2-neighbours.

Rule 2. A $(\geq 4)$-vertex sends $\alpha_{4}$ to its 2-neighbours which are in a 2-thread and $\alpha_{4}^{\prime}$ to its other 2-neighbours.

Let us now define constraints to ensure that the final charge $w^{*}(v)$ of every vertex $v$ of $H$ is at least $M$, a contradiction. Again there are no $(\leq 1)$-vertices by Lemma 1 . 1 .

- Suppose that $v$ is a 2-vertex. Then by Lemma 113 it has at most one 2-neighbour. If $v$ has one 2-neighbour then, by Lemma 1, 4, it has a $(\geq 4)$-neighbour from which it receives $\alpha_{4}$. We obtain the constraint $M \leq 2+\alpha_{4}$. If $v$ has no 2-neighbour then, by Lemma 1 1 , it has a ( $\left.\geq 4\right)$-neighbour from which it receives $\alpha_{4}^{\prime}$. Its other neighbour is a ( $\left.\geq 3\right)$-neighbour from which it receives $\alpha_{3}$. Hence we get $M \leq 2+\alpha_{4}^{\prime}+\alpha_{3}$.

- If $v$ is a 3 -vertex it sends at most $\alpha_{3}$ to each neighbour. This yields the constraints $M \leq 3-3 \alpha_{3}$.

- If $v$ is a 4-vertex then by Lemma 1 7, it is incident to no 2-thread, yielding $M \leq 4-4 \alpha_{4}^{\prime}$.

- If $v$ is a 5-vertex then by Lemma $1 \| 8$, it is incident to at most four 2-threads, yielding $M \leq$ $5-4 \alpha_{4}-\alpha_{4}^{\prime}$ (again we implicitly assume $\alpha_{4} \geq \alpha_{4}^{\prime}$ which is satisfied by the solution giving the optimal value). 
- If $v$ is a $k$-vertex with $k \geq 6$, it sends at most $\alpha_{4}$ to each neighbour. Thus $M \leq k-k \alpha_{4}$.

The optimal value $M=\frac{48}{19}$ is obtained for $\alpha_{3}=\frac{3}{19}, \alpha_{4}=\frac{10}{19}$ and $\alpha_{4}^{\prime}=\frac{7}{19}$.

3 Let $G$ be a graph with maximum degree $\Delta \geq 5$ such that $\operatorname{Mad}(G)<M$. Set $k_{2}=\left\lceil\frac{\Delta}{2}\right\rceil+2$. Suppose by way of contradiction that $\Lambda^{l}(G)>k_{2}$. Then $G$ has a subgraph $H$ which is $k_{2}$-linearminimal.

We assign to every vertex of $H$ an initial charge $w(v)=\operatorname{deg}_{H}(v)$ and apply the following discharging rules.

Rule 1. A 3-vertex sends $\alpha_{3}$ to each of its 2-neighbours

Rule 2. A $(\geq 4)$-vertex sends $\alpha_{4}$ to each of its 2-neighbours having no $(\leq 3)$-neighbour.

Rule 3. A $(\geq 4)$-vertex sends $\alpha_{4}^{\prime}$ to each of its 2-neighbours having a 3-neighbour.

Let us now define constraints to ensure that the final charge $w^{*}(v)$ of every vertex $v$ of $H$ is at least $M$, which is a contradiction.

- If $v$ is a 2-vertex then it has no 2-neighbour by Lemma 111. If it has a 3-neighbour its other neighbour is a $(\geq 4)$-neighbour according to Lemma 1 , yielding $M \leq 2+\alpha_{3}+\alpha_{4}^{\prime}$. If $v$ has no 3-neighbour, then we get $M \leq 2+2 \alpha_{4}$.

- If $v$ is a 3 -vertex it sends at most $3 \alpha_{3}$, yielding $M \leq 3-3 \alpha_{3}$.

- If $v$ is a 4-vertex then by Lemma 10 it has at most one 2-neighbour that has a 3-neighbour, yielding $M \leq 4-\alpha_{4}^{\prime}-3 \alpha_{4}$ (with the assumption $\alpha_{4}^{\prime} \geq \alpha_{4}$ ).

- If $v$ is a $k$-vertex with $k \geq 5$, it sends at most $k \alpha_{4}^{\prime}$, yielding $M \leq k-k \alpha_{4}^{\prime}$.

The optimal value $M=\frac{60}{23}$ is obtained for $\alpha_{3}=\frac{3}{23}, \alpha_{4}=\frac{7}{23}$ and $\alpha_{4}^{\prime}=\frac{11}{23}$.

4 Let $G$ be a graph such that $\operatorname{Mad}(G)<M$. Set $k_{3}=\left\lceil\frac{\Delta}{2}\right\rceil+3$. Suppose by way of contradiction that $\Lambda^{l}(G)>k_{3}$. Then $\Delta \geq 3$, as every graph with maximum degree at most 2 is linearly 3 -choosable. Moreover $G$ has a subgraph $H$ which is $k_{3}$-linear-minimal.

Let us assign to every vertex of $H$ an initial charge $w(v)=d_{H}(v)$ and apply the following discharging rule.

Rule 1. A $(\geq 4)$-vertex sends $\alpha_{4}$ to each of its 2-neighbours

Let us now define constraints to ensure that the final charge $w^{*}(v)$ of every vertex $v$ of $H$ is at least $M$, which is a contradiction.

- If $v$ is a 2 -vertex then it has no $(\leq 3)$-neighbour by Lemmas 111 and 12 . This gives $M \leq$ $2+2 \alpha_{4}$.

- If $v$ is a 3-vertex then its charge does not change, yielding $M \leq 3$.

- If $v$ is a 4 -vertex then by Lemma 1, 13 , it has at most three 2 -neighbours. This yields $M \leq$ $4-3 \alpha_{4}$.

- If $v$ is a $k$-vertex with $k \geq 5$, it sends at most $\alpha_{4}$ to each neighbour, yielding $M \leq k-k \alpha_{4}$.

The optimal value $M=\frac{14}{5}$ is obtained for $\alpha_{4}=\frac{2}{5}$.

5 Let $G$ be a graph such that $\operatorname{Mad}(G)<M$. Set $k_{4}=\left\lceil\frac{\Delta}{2}\right\rceil+4$. Suppose by way of contradiction that $\Lambda^{l}(G)>k_{4}$. Then $\Delta \geq 3$, as every graph with maximum degree at most 2 is linearly 3 -choosable. Moreover $G$ has a subgraph $H$ which is $k_{4}$-linear-minimal. 
Let us assign to every vertex of $H$ an initial charge $w(v)=\operatorname{deg}_{H}(v)$ and apply the following discharging rule.

Rule 1. A ( $\geq 4)$-vertex sends $\alpha_{4}$ to each of its 2-neighbours.

Let us now define constraints to ensure that the final charge $w^{*}(v)$ of every vertex $v$ of $H$ is at least $M$, which is a contradiction.

- If $v$ is a 2-vertex then it has no ( $\leq 3)$-neighbour by Lemmas 111 and 1 12. This gives $M \leq$ $2+2 \alpha_{4}$.

- If $v$ is a 3-vertex, its charge is unchanged. This gives $M \leq 3$.

- If $v$ is a 4-vertex then by Lemma1 14 it has at most two 2-neighbours, yielding $M \leq 4-2 \alpha_{4}$.

- If $v$ is a 5 -vertex then by Lemma 1 15 , it has at most four 2-neighbours. This gives $M \leq$ $5-4 \alpha_{4}$.

- If $v$ is a $k$-vertex with $k \geq 6$, it sends at most $k \alpha_{4}$. Thus $M \leq k-k \alpha_{4}$.

The optimal value $M=3$ is obtained for $\alpha_{4}=\frac{1}{2}$.

\subsection{2-frugal colouring}

In this subsection we prove the following theorem.

Theorem 4. Let $G$ be a graph with maximum degree (at most) $\Delta$

1. If $\Delta \geq 7$ and $\operatorname{Mad}(G)<\frac{5}{2}$, then $\Phi_{2}^{l}(G) \leq\left\lceil\frac{\Delta}{2}\right\rceil+1$.

2. If $\operatorname{Mad}(G)<3$, then $\Phi_{2}^{l}(G) \leq\left\lceil\frac{\Delta}{2}\right\rceil+3$.

Démonstration. 1. Let $G$ be a graph with maximum degree $\Delta \geq 7$ such that $\operatorname{Mad}(G)<M$. Set $k_{1}=\left\lceil\frac{\Delta}{2}\right\rceil+1 \geq 5$. Suppose by way of contradiction that $\Phi_{2}^{l}(G)>k_{1}$. Then $G$ has a subgraph $H$ which is $k_{1}$-frugal-minimal.

Let us assign to every vertex of $H$ an initial charge $w(v)=d_{H}(v)$. Then $\sum_{v \in V(H)} w(v)=\sum_{v \in V(H)} d(v)=$ $\operatorname{Ad}(H) \cdot|V(H)|$. Let us call a 2-vertex bad if it has a 2-neighbour, and $\operatorname{good}$ otherwise. We now apply the following discharging rules.

Rule 1. 3-vertices give $\alpha_{3}$ to each of their 2-neighbours.

Rule 2. ( $\geq 4$ )-vertices give $\alpha_{4}^{g}$ to each of their good 2-neighbours.

Rule 3. ( $\geq 4$ )-vertices give $\alpha_{4}^{b}$ to each of their bad 2-neighbours.

Let us now define constraints to ensure that the final charge $w^{*}(v)$ of every vertex $v$ of $H$ is at least $M$, which is a contradiction

- There are no 1-vertices by Lemma1 1.1 .

- If $v$ is a 2-vertex then by Lemma 13 it does not have two 2-neighbours. In addition, by

Lemma 14 4 no bad vertex can have a 3-neighbour, and by Lemma 11 9 a 2-vertex has at most one

3-neighbour. This gives the constraints $M \leq 2+\alpha_{3}+\alpha_{4}^{g}$ and $M \leq 2+\alpha_{4}^{b}$.

- If $v$ is a 3-vertex it sends at most $\alpha_{3}$ to each of its neighbours, yielding $M \leq 3-3 \alpha_{3}$.

- If $v$ is a 4-vertex, then by Lemma 2 i it has at most one bad neighbour. Hence $M \leq 4-\alpha_{4}^{b}-3 \alpha_{4}^{g}$ (with the assumption $\alpha_{4}^{b} \leq \alpha_{4}^{g}$ ).

- If $v$ is a $k$-vertex with $k \geq 5$ it sends at most $\alpha_{4}^{b}$ to each of its neighbours (under the same assumption), yielding $M \leq k-4 \alpha_{4}^{b}$. 
The optimal value $M=\frac{5}{2}$ is obtained for $\alpha_{3}=\frac{1}{6}, \alpha_{4}^{g}=\frac{1}{3}$ and $\alpha_{4}^{b}=\frac{1}{2}$.

2 Let $G$ be a graph such that $\operatorname{Mad}(G)<M$. Set $k_{3}=\left\lceil\frac{\Delta}{2}\right\rceil+3$. Suppose by way of contradiction that $\Phi_{2}^{l}(G)>k_{3}$. Then $G$ has a subgraph $H$ which is $k_{1}$-frugal-minimal.

We assign to every vertex of $H$ an initial charge $w(v)=\operatorname{deg}_{H}(v)$ and apply the following discharging rule.

Rule 1. $\geq 4$-vertices give $\alpha_{4}$ to each of their 2-neighbours.

Let us now define constraints to ensure that the final charge $w^{*}(v)$ of every vertex $v$ of $H$ is at least $M$, which is a contradiction.

- There are no 1-vertices by Lemma1 1 .

- If $v$ is a 2-vertex, by Lemma 11 it does not have 2-neighbours, and by Lemma 12 it can not have any 3-neighbour either. Hence $M \leq 2+2 \alpha_{4}$.

- If $v$ is a 3 -vertex, $M \leq w^{*}(v)=3$.

- If $v$ is a 4-vertex, by Lemma 2 liil it has at most one 2-neighbour, yielding $M \leq 4-\alpha_{4}$.

- If $v$ is a 5-vertex, by Lemma 2 iiii it has at most four 2-neighbours, giving $M \leq 5-4 \alpha_{4}$.

- If $v$ is a $k$-vertex with $k \geq 6$, then it sends at most $\alpha_{4}$ to each of its neighbours. This yields $M \leq 6-6 \alpha_{4}$.

The optimal value $M=3$ is obtained for $\alpha_{4}=\frac{1}{2}$.

\section{Références}

[1] L. Esperet, M. Montassier and A. Raspaud. Linear choosability of graphs. Discrete Math. 308(17) : 3938-3950, 2008.

[2] B. Grünbaum, Acyclic colourings of planar graphs. Israel J. Math. 14 :390-408, 1973.

[3] H. Hind, M. Molloy, and B. Reed. Colouring a graph frugally. Combinatorica 17 :469-482, 1997.

[4] R. Yuster. Linear colouring of graphs. Discrete Math. 185 :293-297, 1998.

[5] A. Raspaud and W. Wang. Linear coloring of planar graphs with large girth. Discrete Mathematics, 309(18) :5678-5686, 2009.

[6] A. Raspaud and W. Wang, Linear colouring of planar graphs Research report 138406 http://www.labri.fr/perso/lepine/Rapports_internes/RR - 138406.ps.gz, 2006. 
Unité de recherche INRIA Sophia Antipolis 2004, route des Lucioles - BP 93 - 06902 Sophia Antipolis Cedex (France)

Unité de recherche INRIA Futurs : Parc Club Orsay Université - ZAC des Vignes 4, rue Jacques Monod - 91893 ORSAY Cedex (France)

Unité de recherche INRIA Lorraine : LORIA, Technopôle de Nancy-Brabois - Campus scientifique 615, rue du Jardin Botanique - BP 101 - 54602 Villers-lès-Nancy Cedex (France)

Unité de recherche INRIA Rennes : IRISA, Campus universitaire de Beaulieu - 35042 Rennes Cedex (France)

Unité de recherche INRIA Rhône-Alpes : 655, avenue de l'Europe - 38334 Montbonnot Saint-Ismier (France)

Unité de recherche INRIA Rocquencourt : Domaine de Voluceau - Rocquencourt - BP 105 - 78153 Le Chesnay Cedex (France) 